\title{
Hard X-ray detection with a gallium phosphide Schottky diode
}

\author{
Alan Owens $^{* a}$, S. Andersson ${ }^{\mathrm{a}}$, R. den Hartog ${ }^{\mathrm{a}}$, F. Quarati ${ }^{\mathrm{a}}$ A. Webb ${ }^{\mathrm{b}}$, E. Welter ${ }^{\mathrm{b}}$

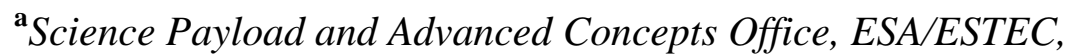 \\ Postbus 299, 2200AG Noordwijk, The Netherlands \\ ${ }^{\mathbf{b}}$ HASYLAB at DESY, Notkestrasse 85, D 22607 Hamburg, Germany
}

\begin{abstract}
We report the detection of hard X-rays using a GaP Schottky diode at the HASYLAB synchrotron radiation research facility. Exposure to alpha particles from an ${ }^{214} \mathrm{Am}$ source showed that the device was spectroscopic at room temperature with a FWHM energy resolution of $3.5 \%$ at $5.5 \mathrm{MeV}$. It was also found to be responsive to X-rays in the range 11-100 keV. Although individual energies are not spectrally resolved there is a proportionality of response to increasing $\mathrm{X}$-ray energy. A two dimensional scan of the sensitive area using a $30 \times 30 \mu \mathrm{m}^{2} 30 \mathrm{keV}$ pencil beam showed the spatial response of the detector to be uniform at the few percent level, consistent with statistics.
\end{abstract}

\section{Introduction}

A number of new materials are being actively developed for sensor needs on present and future ESA space missions. Much of the research has centered on compound semiconductors which offer the ability to operate in a range of chemical, thermal and radiation environments - attributes which can have significant impacts on spacecraft resources and management. However, while compound materials are used routinely in the optical and infrared wave bands, their development at hard X- and $\gamma$ ray wavelengths has been plagued by material and fabrication problems. As part of a comprehensive materials screening program, we have assessed the potential of gallium phosphide as an X-ray detection medium.

Gallium phosphide is an indirect wide bandgap (2.26 eV) semiconductor with a cubic (zincblende) crystal structure. Its density is $4.1 \mathrm{~g} \mathrm{~cm}^{-3}$ - intermediate between Si and Ge. Very little information is available on its transport properties. It was originally investigated as an optical material and for high temperature component applications. However, its main use since the 1960s has been in the manufacture of low and standard brightness red, orange, and green light-emitting diodes. Quite early on it was identified as a possible candidate as a room temperature gamma-ray detection medium [1]. However, this application is unlikely to be realized, given gallium phosphides low electron and hole mobilites $\left(<200 \mathrm{~cm}^{-2} \mathrm{~V}^{-1} \mathrm{~s}^{-1}\right)$ coupled with

* Corresponding author 
the fact that carrier lifetimes in III-V materials rarely exceed 100 ns. Most recently, Litovchenko et al. [2] noted that the IV characteristics of GaP LEDs are permanently altered in strong neutron and electron radiation fields making them candidates for some dosimetry applications.

\section{The detector}

The detector in centered on a commercially available Schottky diode manufactured by the Hamamatsu Corporation, described as a UV to visible photosensor [3]. Its structure consists of a $10 \mathrm{~nm}$ thick Au Schottky layer (anode) deposited on a $30 \mu \mathrm{m} \mathrm{n}$ type GaP layer $\left(\mathrm{n}_{\mathrm{d}}<10^{16} \mathrm{~cm}^{-3}\right)$ grown on an n-type GaP (100) substrate $\left(\mathrm{n}_{\mathrm{d}} \sim\right.$ $5 \times 10^{17} \mathrm{~cm}^{-3}$ ). A further metallization forms the rear contact (cathode). It is packaged in an open TO5 metal housing which is covered with a $5.9 \mathrm{~mm}$ diameter, $1 \mathrm{~mm}$ thick quartz glass window (see figure 1, left). The window serves two functions. It hermetically seals the can and allows the transmission of light to the photosensitive surface. For the experiments described here the window was removed. A Nomarski photomicrograph of this surface is shown in fig. 1 (right). The inner dark brown area
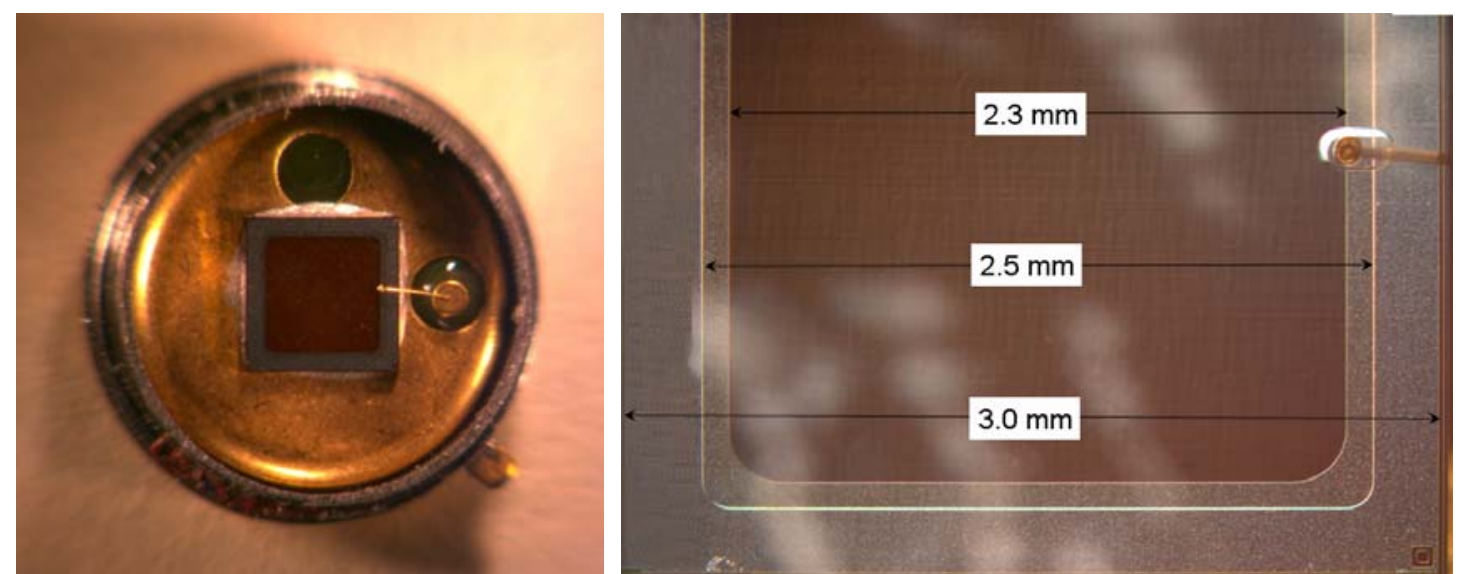

Fig. 1. Left: photograph of the detector. Right Nomarski photomicrograph of the chip. The bond wire is clearly visible at the center of the right hand side. The inner edge of the outer thick metallization defines the low energy X-ray sensitive region $\left(2.5 \times 2.5 \mathrm{~mm}^{2}\right)$ and the inner thin metallization defines the optically sensitive region $\left(2.3 \times 2.3 \mathrm{~mm}^{2}\right)$.

is presumably the optical surface - since it has dimensions $2.3 \times 2.3 \mathrm{~mm}^{2}$ [3]. Later Xray microbeam mapping revealed that the active $\mathrm{X}$-ray area actually extends to $3.0 \times$ $3.0 \mathrm{~mm}^{2}$. The chip is attached to the die by silver epoxy. Thus, the cathode is electrically connected to the case. A bond wire is clearly visible in the center of the right hand side of the chip which terminates on the anode pin out. The current-voltage characteristics were measured as a function of temperature for both forward and reverse biasing using a Keithly Sourcemeter (model no. 2602). The results are shown in fig. 2. The measurements were limited to the range $10 \mathrm{~V}$ to $-30 \mathrm{~V}$. Below $-30 \mathrm{~V}$ the behavior of the diode became erratic. The reverse characteristic shows a small variation for temperatures ranging from $\sim-40^{\circ} \mathrm{C}$ to $+21^{\circ} \mathrm{C}$. From the curves, the resistivity of the diode was estimated to be a few times $10^{10} \Omega \mathrm{cm}$. The inset of fig. 2 shows the corresponding variation of the forward characteristics which is more 
pronounced. The turn-on voltage ranges from $0.3 \mathrm{~V}$ at room temperature to $0.5 \mathrm{~V}$ at $38^{\circ} \mathrm{C}$. The reverse breakdown voltage is $-48 \mathrm{~V}$. C-V measurements were also carried out at room temperature. A plot of $1 / C^{2}$ versus $V$ gives a constant slope, indicating that the diode does not fully deplete.

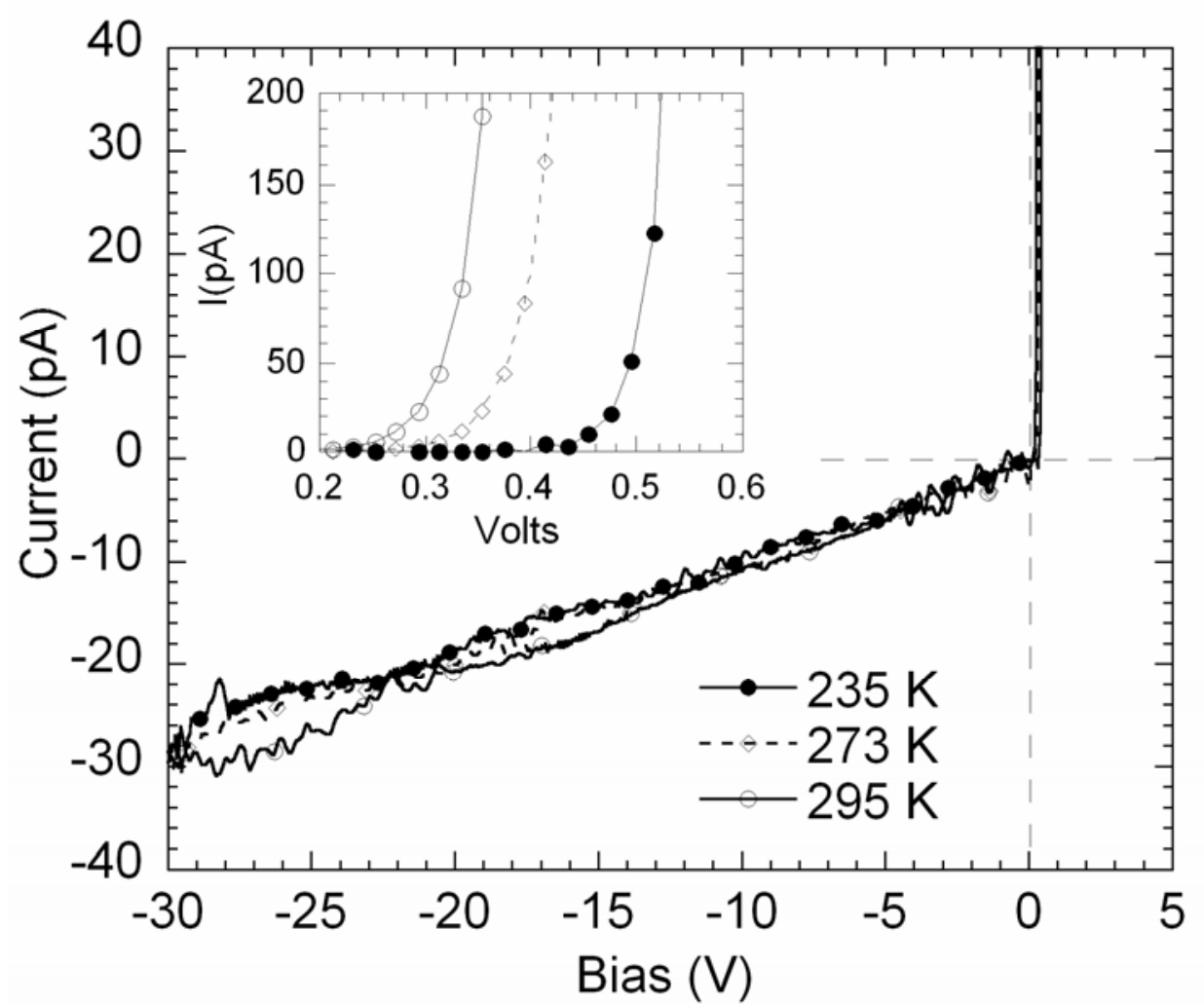

Figure 2. I-V characteristic of the diode at $-38^{\circ} \mathrm{C}, 0^{\circ} \mathrm{C}$ and $21^{\circ} \mathrm{C}$. The inset shows an expansion of the forward characteristic as a function of temperature.

For the experiments described here, the applied bias was set at $+30 \mathrm{~V}$. At this bias, the leakage current is $30 \mathrm{pA}$, which should be low enough to ensure that the device is sensitive at X-ray wavelengths. The depletion depth can be estimated from;

$$
x_{d}=\sqrt{\frac{2 \cdot \varepsilon_{r} \varepsilon_{o}\left(V_{o}+V\right)}{e \cdot n_{d}}}
$$

where $\varepsilon_{r} \cdot \varepsilon_{o}$ is the permittivity of $\mathrm{GaP}, n_{d}$ is the donor concentration, $e$ is the electronic charge, $V_{o}$ is the Schottky barrier height $(\sim 1.35 \mathrm{eV})$ and $V$ is the bias voltage. For the values expected for the diode, coupled with the results of the capacitance measurements, we estimate the depletion depth to be $20 \mu \mathrm{m}<x_{d}<30 \mu \mathrm{m}$.

The diode is mounted on a two stage Peltier cooler capable of cooling the device to $-20^{\circ} \mathrm{C}$. The anode is connected to a resistive feedback charge sensitive preamplifier. The entire assembly is hermetically sealed in a test cryostat. X-rays are viewed through a thin $(25 \mu \mathrm{m})$, vacuum tight beryllium window. The rest of the analog chain consists of an Ortec 671 spectroscopy amplifier whose output is digitized by an Amptek MCA8000A 12-bit ADC and stored on a PC. An amplifier shaping time of $10 \mu \mathrm{s}$ was found to give optimum signal to noise. 
Initial testing of the device was carried out using an ${ }^{241} \mathrm{Am}$ alpha source. We calculate the range of $5.5 \mathrm{MeV}$ alpha particles to be $20 \mu \mathrm{m}$ and therefore they should be contained in the estimated $20 \mu \mathrm{m}$ active layer. The response is shown in fig. 3 from which we can see that the principal peak is clearly resolved. The width is $3.5 \%$ FWHM. Assuming that all charge is indeed fully contained within the active region, the Charge Collection Efficiency (CCE) can be estimated by plotting the peak channel, $P H$, against $1 /$ Bias. The CCE at a given bias is then given by, $C C E(V)=$ $\mathrm{PH} / \mathrm{PH}_{\max }$, where $\mathrm{PH}_{\max }$ is the channel number obtained by extrapolating the linear part of the curve to infinite field (i.e., at $1 / \mathrm{V}=0$ ). At the nominal bias voltage (30 V) the CCE was found to be $95 \%$.

Tests also confirmed a response to $60 \mathrm{keV}$ X-rays from the same source, although non-spectroscopic in this case.

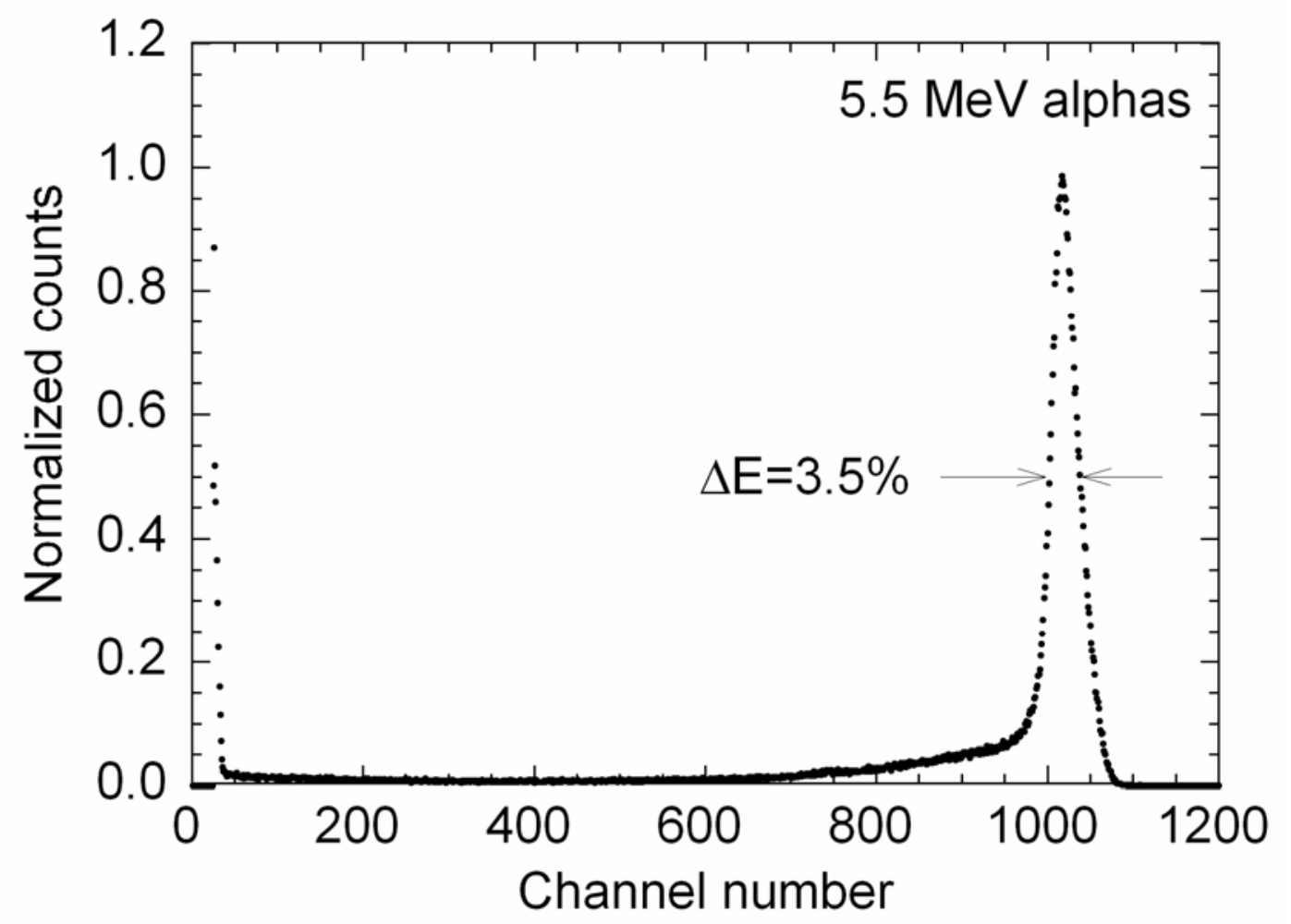

Fig. 3. Measured detector response to $5.5 \mathrm{MeV}$ alpha particles from an ${ }^{241} \mathrm{Am}$ source at room temperature. The width of the line is 3.5\% FWHM.

\section{Synchrotron X-ray measurements}

X-ray characterization was carried out at the $\mathrm{X}-1$ beamline at the Hamburger Synchrotronstrahlungslabor (HASYLAB) radiation facility in Hamburg, Germany [4]. This beamline utilizes a double $\mathrm{Si}$ crystal monochromator to produce highly monochromatic X-ray beams across the energy range $11 \mathrm{keV}$ to $100 \mathrm{keV}$. To achieve such a large energy range, a [511] reflection was used, yielding an intrinsic energy resolution of $\sim 1 \mathrm{eV}$ at $11 \mathrm{keV}$ rising to $20 \mathrm{eV}$ at $100 \mathrm{keV}$. The beam spot size was set by a pair of precision stepper-driven slits, positioned immediately in front of the 
detector. The beamline employs a MOSTAB (MOnochromator STABilizer) servo loop system to ensure constant beam intensity at the detector. The detector was mounted on an X-Y table capable of positioning it to a precision of $<1 \mu \mathrm{m}$ in each axis with respect to the beam. For the measurements described here the beam was normally incident at the center of the forward face of the detector. For the majority of the measurements, a slit size of $50 \times 50 \mu \mathrm{m}^{2}$ was used. Initial noise tests carried out when cooling the device to $-10^{\circ} \mathrm{C}$ resulted in a very slight reduction in noise. Therefore, all subsequent measurements were carried out at room temperature for simplicity. For completeness, additional full-area illumination measurements were also carried out using a ${ }^{109} \mathrm{Ag}$ fluorescent target source.

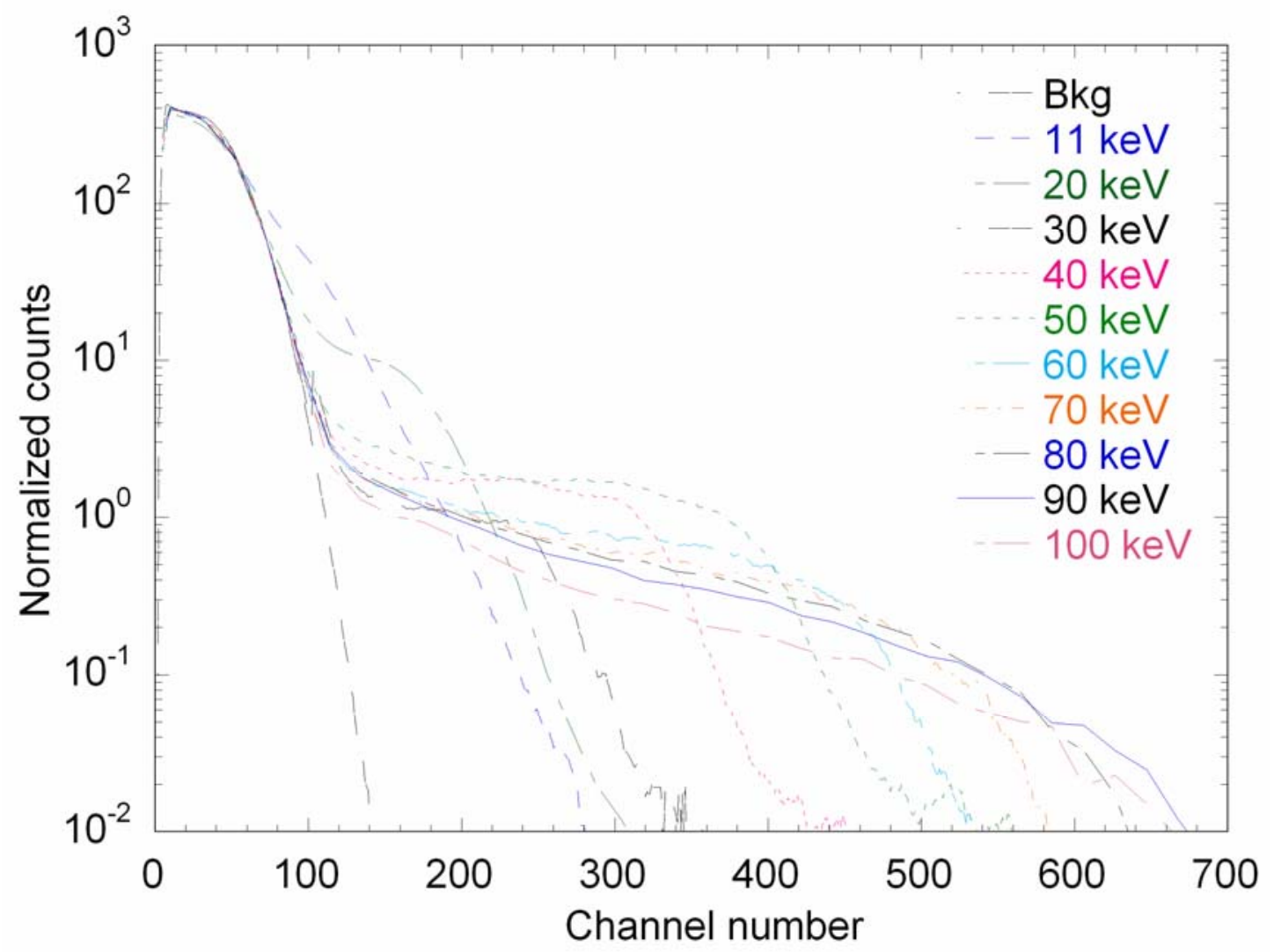

Fig. 4. Composite of energy-loss spectra measured at the center of the detector at room temperature.

In fig. 4 we show a composite of energy loss spectra measured from $11 \mathrm{keV}$ to $100 \mathrm{keV}$. The response shows a strong noise peak and although individual energies are not spectrally resolved there is a proportionality of response to increasing X-ray energy.

The spatial response of the diode was investigated by raster scanning a $30 \mathrm{keV}, 30$ $\times 30 \mu^{2} \mathrm{X}$-ray across the active area in $45 \mu \mathrm{m}$ steps. At each point a spectrum was accumulated for 2.5 seconds. For each spectrum, the total number of counts above the noise peak ( $15 \mathrm{keV}$ ) was recorded and is shown in fig. 5. From the figure, we note that the overall spatial response is uniform at the few percent level, which is consistent with statistics. Surprisingly, the detectors actual sensitive area was found to be $2.5 \times$ $2.5 \mathrm{~mm}^{2}$ with an attenuated response extending out to $3.0 \times 3.0 \mathrm{~mm}^{2}$. Inspection of 
the Nomarski photomicrograph (see figure 1, right) showed that the chip size is $3.0 \times$ $3.0 \mathrm{~mm}^{2}$. There is an outer thick metallization border which is 250 microns wide and a further thin metallization layer extending inwards by 100 microns. The inside dimensions of the thick metallization layer are thus $2.5 \times 2.5 \mathrm{~mm}^{2}$ which corresponds the X-ray sensitive area. The inside dimensions of the thin metallization layer are 2.3 $\times 2.3 \mathrm{~mm}^{2}$, which are the dimensions of the optically sensitive surface. From comparison with the Nomarski image in fig. 1, we can clearly see the attenuation in the thick metallization border and also in the bond wire at the center of the right hand side of the image.
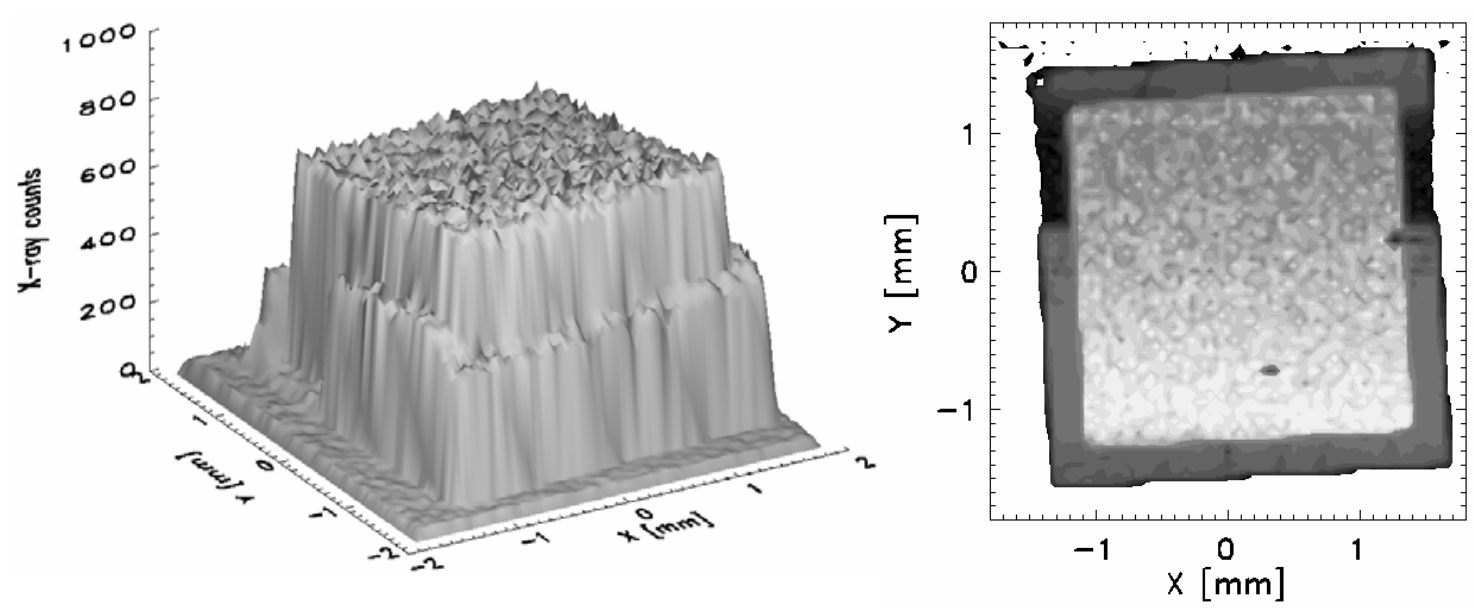

Fig. 5. Left: the spatial variation in the X-ray count rate measured across the detector. The incident Xray energy is $30 \mathrm{keV}$. Right: a contour plot of the X-ray response.

\section{Discussion and conclusions}

We believe this is the first time that a demonstrated direct X-ray response has been shown for GaP. While these results can be considered encouraging, the measured responses can hardly be considered spectroscopic. However, they are unique for each input energy.

This work was supported by the IHP-Contract II-20060238 EC of the European Commission.

\section{References}

[1] L.R. Weisberg, B. Goldstein, GaAs and GaP for room temperature gamma-ray counters, Nucleonics in Aerospace. P. Polishuk, (ed.). New York, Plenum Press (1968) pp 182-186.

[2] P. Litovchenko, D. Bisello, A. Litovchenko, S. Kanevskyj, V. Opilat, M. Pinkovska, V. Tartachnyk, R. Rando, P. Giubilato, V. Khomenkov, Some features of current-voltage characteristics of irradiated GaP light diodes, Nucl. Instr. and Meth., A552 (2005) pp 93-97.

[3] http://www.sales.hamamatsu.com/assets/pdf/parts_G/G1962.pdf

[4] http://www-hasylab.desy.de/ 